\title{
Timing of Cord Clamping and It's Effect on Haematocrit and Clinical Outcome of Neonate
}

\author{
UJJAL MITRA $^{1}$, MD. SHAHIDULLAH ${ }^{2}$, ABDUL MANNAN $^{3}$, ZAKIANAHAR $^{4}$, SANJOY KUMAR DEY ${ }^{5}$, \\ ISTIAQ MANNAN ${ }^{6}$
}

\begin{abstract}
Background: It is the usual practice to clamp umbilical cord immediately after birth. There is no sound evidence to support this approach, which might deprive the newborn of some benefits, such an increase in iron storage.

Objectives: The study was done to determine the effect of timing of cord clamping on neonatal venous haemoglobin and haematocrit values and clinical outcome within neonatal period.

Methodology: This was a randomized, controlled trial performed in Obstetrical Unit with support of Neonatal Unit of Paediatrics and Department of Clinical Pathology of BSMMU on neonates born at term without complication to mothers with uneventful pregnancies. After obtaining written parental consent newborns were randomly assigned to cord clamping within first 15 seconds (group-I Early Cord Clamping, ECC), at around 1 minute (group-II Late Cord Clamping, LCC) after birth. The infant's venous haemoglobin and haematocrit values were measured at 6 hours and 24 to 48 hours after birth.
\end{abstract}

Results: 130 mothers were selected for study purpose. Finally 98 were analyzed, 50 in early cord clamping group and 48 in late cord clamping group. Mean venous haemoglobin and haematocrit values at around 6 and 24 to 48 hours of life were measured. Results were within physiological limit but difference were significant between 2 study groups. The prevalence of anaemia $(\mathrm{Hb}<14 \mathrm{gm} \%)$ was not significantly higher in group-I than group-II but relatively more newborns were anaemic in group-l. There was no significant difference in other neonatal outcomes and maternal postpartum events.

Conclusions: Delayed cord clamping at birth increases neonatal venous haemoglobin and haematocrit values within a physiologic range. No harmful effects were observed among both groups. Furthermore this intervention seems to reduce the incidence of neonatal anemia. This practice has been shown to be safe and should be practiced to increase neonatal haemoglobin and haematocrit values at birth.

Key words: Early cord clamping (ECC), late cord clamping (LCC).

\section{Introduction}

Timing of umbilical cord clamping has been and still is a highly controversial issue ${ }^{1}$. The current obstetric approach in western medicine is to clamp the cord within the first 10 to 15 seconds after birth ${ }^{2}$. However, there has been no sound evidence in favor of this approach in comparison to the millennial practice of clamping the cord between 1 and 3 minutes after

1. Junior Consultant, Paediatrics, Netrakona

2. Professor, Newborn Division, BSMMU, Dhaka

3. Associate Professor, Newborn Division, BSMMU, Dhaka

4. Lecturer, Dhaka Medical College, Dhaka

5. Junior Consultant, Paediatrics, Dohar, Dhaka

6. ICDDRB, Dhaka

Correspondence: Dr. Ujjal Mitra birth $^{3,4}$. In some studies it was observed that delayed cord clamping could contribute to preventing irondeficiency anemia in the first year of life ${ }^{5,6}$. A recent systematic review confirms the benefit of delayed cord clamping. The reason for this effect is based on the fact that after birth the newborn is delivered with a placental transfusion of $80 \mathrm{~mL}$ of blood at 1 minute after birth and $100 \mathrm{~mL}$ at 3 minutes after birth $4,7,8$. This volume will supply 40 to $50 \mathrm{mg} / \mathrm{kg}$ of extra iron to the $75 \mathrm{mg} / \mathrm{kg}$ of body iron that newborn term infants have, reaching a total of 115 to $120 \mathrm{mg} / \mathrm{kg}$, which might prevent iron deficiency in the first year of life ${ }^{2,6}$. Iron deficiency early in life may have pronounced central nervous system effects such as cognitive 
impairment ${ }^{9}$. Iron deficiency is also the main cause of anemia, one of the most serious conditions in childhood, especially in developing countries ${ }^{10}$. Observational study suggests that delayed umbilical cord clamping puts newborns at higher risk of suffering from polycythemia, respiratory symptoms, hyperbilirubinemia, and other neonatal disorders ${ }^{11}$. However, there have been no randomized, controlled trials showing the risk of these harmful effects on the newborn.

In view of the above considerations, we performed this prospective randomized, controlled clinical trial to determine the benefits and risks of delayed cord clamping.

\section{Materials and Methods}

This was a randomized controlled trial performed in BSMMU, in the Department of Gynecology and Obstetrics with collaboration of Neonatal Unit of Paediatrics and Clinical Pathology as a joint protocol. Ethical committee of Department of Gynecology \& Obstetrics and Department of Paediatrics approved this protocol. Finally informed written consent was taken from all participant parents after full explanation of the nature and purpose of the study.

One hundred and thirty mothers were selected for study purpose. At the time of random assignment 10 mothers did not meet the inclusion criteria. Five of the remaining 120 mothers did not agree to participate in the study. The remaining 115 mothers are randomly assigned to the two approaches described for umbilical cord clamping group. Four mothers in the early cord clamping group and 3 mothers in the late cord clamping groups were excluded due to problems of the baby during delivery. Among the rest 108 mothers, 10 missed the follow up. So, finally 98 were analyzed, 50 in Group-I, early cord clamping (ECC) group and 48 in Group-II, late cord clamping (LCC) group.

Full term newborn with uneventful antenatal history were the candidate for study. Exclusion criteria were maternal diabetes, preeclampsia, hypertension, severe maternal anemia $(\mathrm{Hb}<7 \mathrm{gm} / \mathrm{d}$ ), IUGR, newborns who needed resuscitation, congenital anomalies and any other complications at birth.

Two interventions, early and late cord clamping for the newborn were compared. Early umbilical cord clamping was defined as clamping within first $15 \pm 5$ seconds after birth and late umbilical cord clamping as clamping at $60 \pm 5$ seconds after birth. The cord clamping technique was similar in both groups by umbilical cord clamp.

Newborns of both groups were placed on a tray after delivery. The tray was held at the level of the introitus by a helping staff. The baby was provided immediate newborn care by drying, wrapping and warming with warm sterile sheets. The newborn was examined clinically. Birth weight, supine length and OFC were measured. Mothers were also followed up by examining vital parameters.

Venous haemoglobin and haematocrit in the newborn was measured in blood drawn from a peripheral vein. Blood was collected in a $\mathrm{CBC}$ vial sealed at one end with the cork. Haemoglobin was measured by automated hematology analyser, by colorimetric principle. Hct was also measured by automated hematology analyser using the principle of immunological staining and flowcytometry. Serum bilirubin was measured by photometric colorimetric test for total bilirubin.

Every newborn included in the study was followed up at 7 and 28 days of life. During these visits weight, supine length and OFC were measured. Feeding patterns were recorded also.

Maternal $\mathrm{Hb} \%$ was measured at 24 hours before and after delivery.

The information collected from the data forms was analysed by using statistical software program for data analysis STATA version 8.0. Means were calculated and compared by using two sample t-test and proportions were measured by using chi-square test. Values were presented as mean, \pm standard deviation. Respective P-value, 95\% confidence interval were considered to determine level of significance.

FLOW CHART OF THE STUDY DESIGN

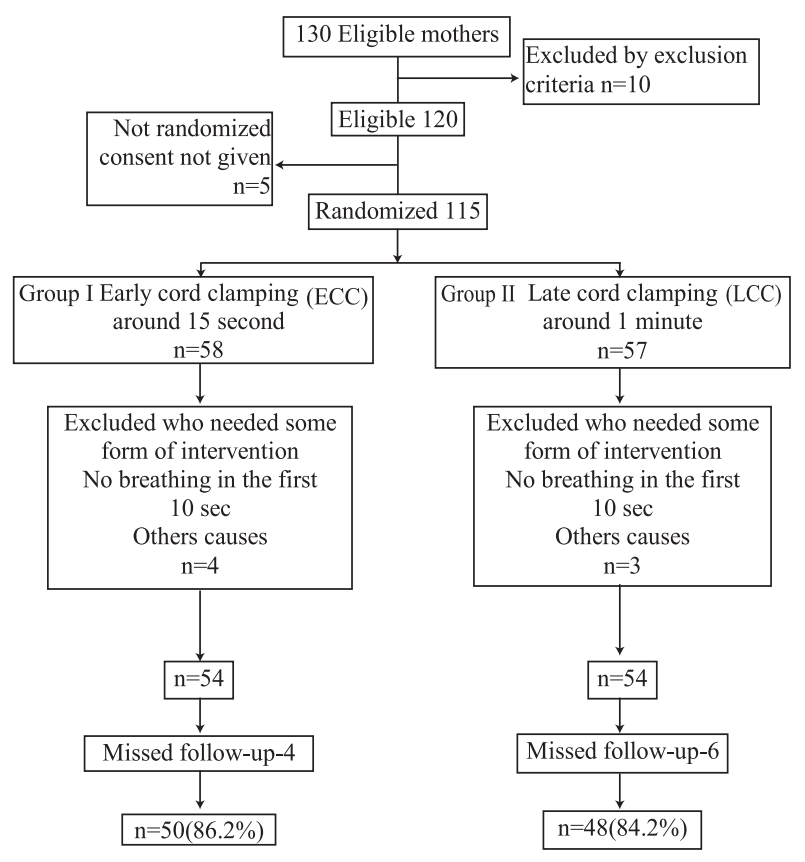




\section{Results}

Among 130 selected pregnant mothers 98 newborns were analyzed, 50 in early cord clamping (ECC) group and 48 in late cord clamping (LCC) group.

Average clamping time for each group was 14 second in ECC group and 58.4 second in late cord clamping LCC group. Study group were similar in demographic and clinical variables (Table-I).

Neonatal haemoglobin and haematocrit at 6 hours of age in ECC group were 16.14 $1.049 .0 \pm 2.9$, and in the late LCC were $17.4 \pm 1.0,53.0 \pm 3.1$ respectively. The difference between early and late group in $\mathrm{Hb} \%$ and haematocrit was highly significant (Table-II).

Neonatal anaemia in ECC group is relatively higher than LCC group at 6 hours of life $(n=4,8.0 \% ; n=1$, $2.1 \%)$ and also at 24 to 48 hours of life $(n=8,16 \%$; $n=3,6.3 \%)$ but the differences were not statically significant (Table-III).

Only one baby in late clamping group developed polycythemia (Hct-65.0\%), but none in early cord clamping group. Plasma bilirubin level was similar in both the groups (Table-IV). Two newborns in LCC group and one in ECC group developed significant jaundice. One in LCC group was due to ABO incompatibility and others were exaggerated physiological jaundice. One newborn in each group developed respiratory distress (Transient Tachypnoea of Newborn, TTN). The clinical course after discharge during the first month of life was similar in both groups. Newborns of both groups were observed for physical growth up to neonatal period. There was no significant difference in weight, supine length and occipitofrontal circumference. Maternal outcome after delivery was also similar in both study groups (Table-V).

Table-I

Baseline characteristics of the study groups before delivery

\begin{tabular}{lccc}
\hline Baseline Characteristics & ECC group & LCC group & p value \\
\hline Maternal age, mean \pm SD $(95 \% \mathrm{Cl})$ & $26.8 \pm 5.4(25.3-28.3)$ & $26.6 \pm 4.4(25.4-28.0)$ & 0.86 \\
Maternal pregnancy BMI, mean \pm SD $(95 \% \mathrm{Cl})$ & $25.3 \pm 1.9(24.7-25.8)$ & $24.9 \pm 2.3(24.2-25.6)$ & 0.33 \\
Primipara, $\mathrm{n} / \mathrm{N}(\%)$ & $24 / 50(48.0)$ & $27 / 48(56.3)$ & 0.40 \\
Normal delivery, $\mathrm{n} / \mathrm{N}(\%)$ & $18 / 50(26.0)$ & $20 / 48(41.7)$ & 0.56 \\
Maternal haemoglobin (before delivery), & $9.8 \pm 1.0(9.5-10)$ & $9.7 \pm 0.8(9.5-10)$ & 0.98 \\
mean $\pm \mathrm{SD}(95 \% \mathrm{Cl})$ & & & \\
Newborn gestational age, mean $\pm \mathrm{SD}(95 \% \mathrm{Cl})$ & $38.8 \pm 1.4(38.4-39.2)$ & $38.6 \pm 1.5(38.0-39.0)$ & 0.47 \\
\hline
\end{tabular}

Table-II

Comparison of neonatal haemoglobin and haematocrit level, mean difference of haematocrit at 6 hours and 24 to 48 hours age both early and late cord clamping group

\begin{tabular}{lccc}
\hline & $\begin{array}{c}\text { ECC group } \\
\text { mean } \pm \text { SD } \\
(95 \% \mathrm{Cl})\end{array}$ & $\begin{array}{c}\text { LCC group } \\
\text { mean } \pm \text { SD } \\
(95 \% \mathrm{Cl})\end{array}$ & p value* \\
\hline Neonatal haemoglobin at 6 hours of age & $16.14 \pm 1.1(15.82-16.4)$ & $17.4 \pm 1.0(17.1-17.7)$ & $0.001^{* *}$ \\
Neonatal haematocrit at 6 hours of age & $48.5 \pm 2.9(47.7-49.4)$ & $52.5 \pm 3.1(51.6-53.5)$ & $0.001^{* *}$ \\
Neonatal haemoglobin at 24 to 48 hours of age & $15.6 \pm 0.9(15.3-15.8)$ & $16.3 \pm 0.9(16.0-16.6)$ & $0.001^{\text {** }}$ \\
Neonatal haematocrit at 24 to 48 hours of age & $46.7 \pm 2.0(46-47.5)$ & $49.4 \pm 3.6(48.4-50.5)$ & $0.001^{* *}$ \\
Mean differences in Hct in 6 hours \& 24 hours & $1.8 \pm 1.5(1.37-2.23)$ & $3.1 \pm 1.7(2.62-3.63)$ & $0.001^{* *}$ \\
\hline
\end{tabular}

* two sample t-test ${ }^{* *}$ significant ( $p$-value 0.001) 
Table-III

Comparison of Neonatal anemia in early and late cord clamping groups

\begin{tabular}{lccccc}
\hline & \multicolumn{2}{c}{ Early } & & \multicolumn{2}{c}{ Late } \\
\cline { 2 - 3 } & No. & $\%$ & & No. & $\%$ \\
\hline $\mathrm{Hb}<14$ gm\% at 6 hours of age & & 4 & 8.0 & & 1 \\
$\mathrm{Hct}<14 \mathrm{gm} \%$ at 24 to 48 hours age $^{\star *}$ & 8 & 16.0 & & 3 & 6.1 \\
\hline
\end{tabular}

${ }^{*} \mathrm{p}$ value $=0.18,95 \% \mathrm{Cl}=0.00-2.6 \mathrm{RR}, .24$

${ }^{* * \mathrm{p}}$ value $=0.13,95 \% \mathrm{Cl} 0.05-1.60 \mathrm{RR}, .35$

Chi-square test

Table-IV

Characteristic of study groups after delivery

\begin{tabular}{lcc}
\hline & ECC group & LCC group \\
\hline Birth weight in gm, mean \pm SD & $2804.6 \pm 211.0$ & $2876.0 \pm 241.4$ \\
$(95 \% \mathrm{Cl})$ & $(2744.6-2864.5)$ & $(2805.9-2946)$ \\
Serum bilirubin level mean \pm SD $(95 \% \mathrm{Cl})$ & $5.3 \pm 1.9(4.7-5.8)$ & $6.2 \pm 4.9(4.8-7.6)$ \\
Serum bilirubin >16mg/dl (n/N\%) & $1 / 50(2 \%)$ & $2 / 48(4.16)$ \\
Respiratory Status & $1 / 50(2 \%)$ & $1 / 48(2.08)$ \\
(Tachypnoea,Distress) & & \\
*Abnormal neonatal outcome after discharge n/N \% & $6 / 15(12 \%)$ & $3 / 48(6.25 \%)$ \\
Infant feeding at 1 month n/N\% & & $31 / 48(47.6)$ \\
\multicolumn{1}{c}{ Exclusive Breast feeding } & $34 / 50(68 \%)$ & $9 / 48(45 \%)$ \\
$\quad$ Partial Breast feeding & $11 / 50(22 \%)$ & $8 / 48(61.54)$ \\
\hline Breast milk substitute & $5 / 50(10 \%)$ & \\
\hline
\end{tabular}

*Diarrhoea, Protracted jaundice, Respiratory tract infection

Table-V

Characteristic of study group mothers after delivery

\begin{tabular}{lcc}
\hline & ECC group & LCC group \\
& Mean \pm SD $(95 \% \mathrm{Cl})$ & Mean \pm SD $(95 \% \mathrm{Cl})$ \\
\hline Maternal $\mathrm{Hb} \%$ after delivery & $9.06 \pm 1.0(8.7-9.5)$ & $9.1 \pm 0.9(8.8-9.5)$ \\
Mean difference of maternal $\mathrm{Hb}$ after delivery & $0.72 \pm 0.26(0.065-0.79)$ & $0.63 \pm 0.44(0.50-0.76)$ \\
\hline
\end{tabular}

\section{Discussion}

Although many controlled trials have not shown any harmful effects related to delayed cord clamping, immediate clamping is still the common practice. Early clamping deprives newborn from large quantities of blood and other benefits. The iron content of the normal newborn infant is approximately $75 \mathrm{mg} / \mathrm{kg}$. Iron stores at birth are variable and varies with $\mathrm{Hb} \%$ or $\mathrm{Hct}$ values of the newborn ${ }^{12}$. Iron level at birth correlates with iron level at 6, 9 and 12 months of age ${ }^{6}$. Dietary iron is one of the factors influencing the iron status during the first year of life ${ }^{13}$. These considerations have influenced the beneficial effect of delayed cord clamping over early cord clamping. This prospective randomized controlled study evaluated the effect of delayed cord clamping on neonatal haemoglobin and Hct values at two different cord clamping time intervals (early cord clamping and late cord clamping). The outcome of the newborns of both study groups were also evaluated at two times ( 7 days and 28 days of 
age) within the neonatal period to observe any neonatal morbidity and mortality. We observed significant increase in haemoglobin and haematocrit values in delayed cord clamping group than early cord clamping group, but values were within physiological limit. These finding correlates with others observation ${ }^{8,14}$. As described in other studies $7,8,15$ no polycythemia related harmful effect were observed in the only polycythemic newborn in delayed cord clamping group. Regarding anemia, anemic newborns were more in ECC group than LCC group both at 6 and 24 to 48 hours of life. Others shows the number significant, as because number of cases were more ${ }^{16}$. Plasma bilirubin values as well as hyperbilirubinemia rates were similar in both groups which is comparable with other authors observation ${ }^{7,12}$. Respiratory disorders were transient (Transient tachypnoea of newborn) and was managed with short time oxygen therapy.

Influence of gravity on placental transfusion is variable. In this study newborn was held at the level of the introitus. An author observed that blood volume of newborn increases if the baby is held $20 \mathrm{~cm}$ below the introitus ${ }^{17}$. But other could not demonstrate any influence of gravity on placental transfusion ${ }^{5}$.

Many authors observed their subject until 2 to 6 month of age and observed the effect of delayed cord clamping on iron status of the baby in early infancy. Result is variable $^{18}$, one observed no difference, others observed significant increase in $\mathrm{Hb} \%$ and serum ferritin level at 3 and 6 months of age ${ }^{19,20}$.

Maternal outcome was also similar in both the study groups. Post partum blood loss was not measured but $\mathrm{Hb} \%$ at around 24 hours before and after delivery were similar in both the study groups (Table-V). These results are in agreement what other authors have reported and refuses the belief that delayed cord clamping is associated with greater postpartum bleeding ${ }^{16}$.

\section{Conclusion}

Clamping of the umbilical cord at 1 minute after birth resulted in significant increase in haematocrit values measured at around 6 hours of age. The average haemoglobin level was above the defined level anaemia. But when individually considered more newborns were anaemic in early cord clamping group than in late cord clamping group.

Delayed cord clamping is not associated with any harmful effects on newborns and may contribute in increasing iron stores, thus averting incidence of iron deficiency anaemia in the first year of life. But to establish this protocol of delayed cord clamping in Bangladesh large scale multicentre studies are needed.

\section{References}

1. Peltonen T. Placental transfusion: advantage and disadvantage. Eur J Pediatr 1981; 137: 141-46.

2. Pisacane A. Neonatal prevention of iron deficiency. BMJ 1996; 312: 136-37.

3. World Health Organization. Care of the Umbilical Cord: A Review of the Evidence. Geneva, Switzerland: World Health Organization; 1998:1112. WHO/RHTMSM/98.4.

4. Mercer JS. Current best evidence: a review of the literature on umbilical cord clamping. J Midwifery Womens Health 2001; 46: 402-14.

5. Grajeda R, Perez-Escamilla R, Dewey KG. Delayed clamping of the umbilical cord improves hematologic status of Guatemalan infants at 2 mo of age. Am J Clin Nutr 1997; 65: 425-31.

6. Michaelsen KF, Milman N, Samuelson G. A longitudinal study of iron status in healthy Danish infants: effects of early iron status, growth velocity and dietary factors. Acta Paediatr 1995; 84: 1035-44.

7. Van Rheenen P, Brabin BJ. Late umbilical cordclamping as an intervention for reducing iron deficiency anaemia in term infants in developing and industrialised countries: a systematic review. Ann Trop Paediatr 2004; 24: 3-16.

8. Linderkamp O, Nelle M, Kraus M, Zilow EP. The effect of early and late cord-clamping on blood viscosity and other hematological parameters in full-term neonates. Acta Paediatr 1992; 81: 745-50.

9. Lozoff B, Jimenez E, Hagen J, Mollen E, Wolf AW. Poorer behavioral and developmental outcome more than 10 years after treatment for iron deficiency in infancy. Paediatrics 2000; 105. Available at: www.paediatrics.org/cgi/content/ full/105/4/e51.

10. Calvo EB, Gnazzo N. Prevalence of iron deficiency in children aged 9-24 mo from a large urban area of Argentina. Am J Clin Nutr 1990; 52: 534-40. 
11. Saigal S, O'Neill A, Surainder Y, Chua LB, Usher R. Placental transfusion and hyperbilirubinemia in the premature. Paediatrics 1992; 49: 406-19.

12. Oski FA. Anemia in Infancy: Iron deficiency and vitamin E deficiency. Paediatrics in Review 1980; 1: 247-53.

13. Stevens $D$, Nelson $A$. The effect of iron in formula milk after 6 months of age. Arch Dis Child 1995; 73: $216-20$.

14. Nelle M, Zilow EP, Kraus M, Bastert G, Linderkamp O. The effect of Leboyer delivery on blood viscosity and other hematologic parameters in term neonates. Am J Obstet Gynecol 1993; 169: 189-93.

15. Oxford Midwives Research Group. Astudy of the relationship between the delivery to cord clamping interval and the time of cord separation. Midwifery 1991; 7: 167-76.
16. Jose M, Cernadas C, Pelligrini L, Otano I, Marina F. The effect of timing of cord clamping on neonatal venous haematocrit values and clinical outcome at term. Pediatrics 2006; 117: 779-86.

17. Yao AC, Lind J. Effect of gravity on plancetal transfusion. Lancet 1999; 65: 425-31.

18. Geethanath R, Ramji S, Thirupuran S, Rao Y. Effect of timing of cord clamping on the iron status of infants at 3 month. Indian Paediatrics 1997; 34: 103-06.

19. Chaparro CM, Neufeld LM, Alavez GT. Effect of timing of umbilical cord clamping on iron status in Mexican infants: a randomized controlled trial. Lancet 2006; 82: 367.

20. Gupta R, Ramji S. Effect of delayed cord clamping on iron stores in infants born to anemic mothers: A randomized controlled trial. Indian Paediatrics 2001; 39: 1-2. 